\title{
U-shaped effect of blood pressure on structural OCT metrics and retinal blood flow autoregulation in ophthalmologically healthy subjects
}

\author{
Konstantinos Pappelis, ${ }^{1,2}$ Nomdo M. Jansonius ${ }^{1,2}$
}

1. Department of Ophthalmology, University of Groningen, University Medical Center Groningen, Groningen, The Netherlands

2. Graduate School of Medical Sciences (Research School of Behavioural and Cognitive Neurosciences), University of Groningen, Groningen, The Netherlands

Correspondence: K. Pappelis, Dept. of Ophthalmology, University Medical Center Groningen, P.O.Box 30.001, 9700 RB Groningen, Netherlands. Phone: +31 50 3612510; Fax: +31 50

3611709; e-mail:k.pappelis@rug.nl

E-mail co-authors: n.m.jansonius@umcg.nl

Running head: Blood pressure and structural OCT: a U-shaped association

Conflict of interest: No conflicting relationships exist for any author.

Financial support: European Union's Horizon 2020 Innovative Training Networks Program, under the Marie Sklodowska - Curie grant, Project ID 675033. The funding organization had no role in the design, conduct, analysis, or publication of this research.

Word count: 4165 (main text); 350 (Abstract)

Meeting presentation: European Association for Vision and Eye Research (EVER); Nice, 3rd-6th October 2018.

Keywords: glaucoma, optical coherence tomography, blood pressure, blood flow, autoregulation

Abbreviations/Acronyms: AHT, arterial hypertension; AR, autoregulatory reserve; BMI, body mass index; BP, blood pressure; CRAE, central retinal artery equivalent; CRVE, central retinal vein equivalent; DBP, diastolic blood pressure; FD, fractal dimension; GCIPL, ganglion cell- 
35 inner plexiform layer; IOP, intraocular pressure; IQR, interquartile range; LARL, lower

36 autoregulation limit; MAP, mean arterial pressure; mRNFL, macular retinal nerve fiber layer;

37 NTG, normal-tension glaucoma; OCT, optical coherence tomography; OCT-A, optical

38 coherence tomography-angiography; $\mathrm{ONH}$, optic nerve head; OPP, ocular perfusion pressure;

39 pRNFL, peripapillary retinal nerve fiber layer; RBF, retinal blood flow; RGC, retinal ganglion cell;

40 RNFL, retinal nerve fiber layer; RPP, retinal perfusion pressure; RVR, retinal vascular

41 resistance; SBP, systolic blood pressure; SD, standard deviation; SEQ, spherical equivalent.

42

43

44

45

46

47

48

49

50

51

52

53

54

55

56

57

58

59

60

61

62

63

64

65

66

67

68 


\section{Abstract}

Objective: 1) To investigate the effect of low blood pressure (BP), treated arterial hypertension

$72(\mathrm{AHT})$, and untreated $\mathrm{AHT}$ on the ganglion cell-inner plexiform layer (GCIPL) and the retinal

73 nerve fiber layer (RNFL) thickness of non-glaucomatous eyes and 2) to elucidate whether this

74 effect is related to crossing the lower limit of retinal blood flow (RBF) autoregulation.

Design: Cross-sectional, case-control.

78 Subjects: We included 96 eyes of 96 ophthalmologically healthy subjects (age 50-65).

79 Participants were prospectively recruited from a large-scale cohort study in the northern

80 Netherlands ( $n=167,000$; Lifelines Biobank). They were allocated to four groups (low BP, normal

$81 \mathrm{BP}$ [controls], treated $\mathrm{AHT}$, untreated $\mathrm{AHT}$ ), based on information from previous visits and strict

82 distribution criteria.

84 Methods: Inner retinal layer thicknesses were obtained with optical coherence tomography

85 (OCT). Fractal dimension of the superficial microvasculature was quantified with OCT-

86 angiography and customized software. Central retinal vessel diameters were obtained from

87 fundus images. BP and intraocular pressure measurements were also acquired. Measurements

88 were combined with a validated physiological model to estimate vascular outcome measures.

89 Structural and vascular metrics were compared across groups and mediation analysis was

90 performed.

91

92 Main outcome measures: Structural: macular GCIPL and RNFL (mRNFL), peripapillary RNFL

93 (pRNFL) thickness. Vascular: RBF, retinal vascular resistance (RVR), autoregulatory reserve

$94(\mathrm{AR})$. 
96 Results: Compared to controls, GCIPL was thinner in the low BP group $(P=0.013)$, treated

97 hypertensives $(P=0.007)$, and untreated hypertensives $(P=0.007)$. Treated hypertensives

98 exhibited the thinnest mRNFL $(P=0.001)$, temporal pRNFL $(P=0.045)$, and inferior pRNFL

$99(P=0.034)$. In multivariable analysis, RBF was mediating the association of GCIPL thickness

100 with BP within the combined low BP group and controls $(P=0.003)$, RVR together with AR were

101 mediating the same association within the combined treated hypertensives and controls

$102(P=0.001$ and $P=0.032)$, and RVR was mediating the association within the combined untreated

103 antihypertensives and controls $(P=0.022)$.

104

105 Conclusions: We uncovered GCIPL and RNFL thinning related to both tails of the BP

106 distribution. GCIPL thinning was associated with reduced RBF autoregulatory capacity. This

107 predisposition to glaucomatous damage could explain the frequent epidemiological finding of

108 increased glaucoma risk in certain subgroups, such as subjects with nocturnal BP dipping or

109 aggressively treated AHT. Longitudinal studies could confirm this postulation. 
medRxiv preprint doi: https://doi.org/10.1101/2021.01.14.21249808; this version posted January 15, 2021. The copyright holder for this preprint (which was not certified by peer review) is the author/funder, who has granted medRxiv a license to display the preprint in perpetuity.

It is made available under a CC-BY-NC-ND 4.0 International license .

\section{Introduction}

Glaucoma is a chronic optic neuropathy characterized by thinning of the retinal nerve fiber layer (RNFL), loss of retinal ganglion cells (RGCs), and progressive visual function decline. ${ }^{1}$ While elevated intraocular pressure (IOP) is considered as the most important modifiable risk factor, glaucoma may also manifest in those with apparently normal IOP (normaltension glaucoma [NTG]). ${ }^{2-4}$ Therefore, certain components of the disease remain elusive or insufficiently addressed.

It has been proposed that low or unstable blood supply could lead to reduced oxygenation of the RGCs. ${ }^{4,5}$ Current assessment of RGC structure is based on Optical Coherence Tomography (OCT), while its extensions, OCT-angiography (OCT-A) and Doppler OCT, enable the non-invasive evaluation of retinal perfusion. ${ }^{6-8}$ These methods have already revealed that reduced blood flow predicts visual field (VF) deterioration, independently of neural tissue damage. ${ }^{9-11}$ However, after the onset of the disease, it is impossible to disentangle if perfusion deficits are the cause (low supply) or consequence (low demand) of glaucomatous optic neuropathy (GON). This realization is known as the 'chicken-egg' dilemma in glaucoma.

From a hemodynamic perspective, blood flow is determined by the balance between ocular perfusion pressure (OPP) and vascular resistance. ${ }^{12}$ Therefore, low blood pressure (BP) could result in low OPP, thus increasing the risk for glaucoma incidence and progression, possibly due to flow-mediated damage to the RGCs. Indeed, this has been observed in some cross-sectional and longitudinal population-based studies. ${ }^{13-16}$ However, other studies do not confirm this finding, while there is also evidence that this association becomes relevant only when low BP manifests as pronounced nocturnal dipping. ${ }^{17,18}$ On the other hand, while arterial hypertension $(\mathrm{AHT})$ is also frequently reported as a risk factor for glaucoma, conflicting results exist on whether BP reduction exacerbates or protects from GON, possibly depending on individual medication effects and on how aggressive the treatment strategy is. ${ }^{19-26}$ 
This study focuses on ophthalmologically healthy subjects, rather than glaucoma subjects. This is essential, because the next logical step in approaching the 'chicken-egg' dilemma is to move back in the disease time course and study the effect of BP on retinal blood flow (RBF) and the RGCs, prior to any glaucoma onset. To investigate RBF, we look into the

151 retinal microcirculation, which demonstrates the ability of autoregulation, i.e., active modification

152 of vascular caliber in response to local signals. ${ }^{27}$ This property protects the tissue from

153 ischemia, in case of OPP drops. Recently, we proposed and validated a microcirculation model

154 predicting which healthy subjects are more prone to hypoperfusion, by estimating their lower

155 autoregulation limit (LARL) through OCT-A, fundus imaging, and other clinical examinations. ${ }^{28}$

156 In the present study, we hypothesized an inverse U-shaped association between BP

157 status and structural OCT measures in non-glaucomatous eyes. While the detrimental effect of

158 AHT to the RGCs and their axons has been previously documented, this effect has not been

159 studied in subjects with low BP, nor has it been examined in combination with RBF

160 autoregulation. ${ }^{29-32}$ Studies until now have used linear models to describe the association

161 between BP and structural OCT measures, thus potentially neglecting any signal coming from

162 the left tail of the distribution. ${ }^{33,34}$

Therefore, the aims of this study were 1) to investigate the effect of low BP, treated AHT,

164 and untreated AHT on the inner retinal layer thicknesses of non-glaucomatous eyes and 2) to

165 elucidate whether this effect is related to crossing the lower limit of RBF autoregulation. For this

166 purpose, we performed multimodal structural and vascular imaging in ophthalmically healthy

167 normotensive controls, treated arterial hypertensives, and individuals belonging to the lower and

168 higher (untreated) tails of the BP distribution. Participants were selected from the large-scale

169 population-based Lifelines cohort, which enabled us to study the real extremes, especially from

170 the low BP tail, in an unbiased manner. 


\section{Methods}

\section{Study design and population}

For this cross-sectional, case-control study, we prospectively recruited subjects via targeted invitation among the participants of a large-scale prospective cohort study of the

179 northern Netherlands (Lifelines Biobank; $n=167,000) .{ }^{35}$ Subjects were invited based solely on

180 their BP status and age. Following a strict selection procedure, 105 participants between 50 and

18165 years of age satisfied both the BP criteria (see next paragraph) and the ophthalmic and

182 medical history inclusion criteria: unoperated eyes; best-corrected visual acuity $\geq 0.8$; spherical

183 refractive error between -3 and $+3 \mathrm{D}$; cylinder not exceeding $2 \mathrm{D}$; IOP $\leq 21 \mathrm{mmHg}$ (non-contact

184 tonometer Tonoref II, Nidek, Aichi, Japan); no reproducibly abnormal VF test locations

185 (Frequency Doubling Technology [C20-1 screening mode], Carl Zeiss, Jena, Germany); no

186 family history of glaucoma; no ophthalmic, hematologic, or cardiovascular disease (except for

$187 \mathrm{AHT}$ ), and no diabetes. We performed additional documentation of ophthalmic health with the

188 subsequent imaging sessions (see Data collection). We allocated participants to four non-

189 overlapping groups: 1) low BP, 2) normal BP (controls), 3) treated AHT, and 4) untreated AHT.

190 Group definitions (see next paragraph) were based on previous epidemiological evidence on the

191 association between BP status and glaucoma. ${ }^{15,16}$ All participants provided written informed

192 consent. The ethics board of the University Medical Center Groningen approved the study

193 protocol (\#NL61508.042.17). The study followed the tenets of the Declaration of Helsinki.

\section{Blood pressure group definitions}

We defined low BP (group 1) as both systolic and diastolic BP (SBP, DBP) lower than 198 the 10th percentiles of the age-matched population (110 $\mathrm{mmHg}$ and $65 \mathrm{mmHg}$, respectively), 
without any AHT record. This criterion had to be confirmed on at least two previous, separate occasions (ascertaining that subjects truly belonged to the tail of the distribution and did not

201 regress towards the mean). We defined untreated AHT (group 4) similarly, the criteria being

202 both SBP and DBP higher than the 90th percentiles of the age-matched population (149 $\mathrm{mmHg}$

203 and $88 \mathrm{mmHg}$, respectively), verified at least twice previously. Subjects of this group were

204 aware of their BP status, but never made use of antihypertensive medication, by choice. For

205 treated AHT (group 3), we randomly invited participants documented as receiving (and still

206 making uninterrupted use of) antihypertensive medication for at least one year. Lastly, we

207 defined normal BP (group 2) as both SBP and DBP within 1 standard deviation (SD) from the

208 mean of the age-matched population (SBP: $113 \mathrm{mmHg}$ to $143 \mathrm{mmHg}$ and DBP: $67 \mathrm{mmHg}$ to 85

$209 \mathrm{mmHg}$, measured on site) and no previous record of AHT.

Data collection

All participants were examined at the same time of the day (5:00 PM-6:30 PM) and were

214 not given any instructions regarding their routine prior to their visit. Following screening (see

215 previous section), we applied mydriatic drops that have been shown to not affect RBF

216 (tropicamide 0.5\%). ${ }^{36}$ After the participants had rested in a quiet room for 20 minutes, we

217 recorded BP from the brachial artery, in sitting position, with an automatic monitor (Omron M6

218 Comfort, Omron Healthcare, Kyoto, Japan). We averaged two readings, unless there was a

219 discrepancy of at least $10 \mathrm{mmHg}$ in SBP or $5 \mathrm{mmHg}$ in DBP, in which case we averaged three

220 readings. We also measured the weight and height of each participant.

221 For the imaging session, we selected, randomly if both eyes fulfilled the inclusion

222 criteria, one eye per participant. We performed macular and optic nerve head $(\mathrm{ONH})$ structural

223 OCT imaging, as well as parafoveal OCT-A (Canon HS100 SD-OCT, Tokyo, Japan). The device

224 automatically segments and quantifies macular RNFL (mRNFL) thickness and ganglion cell- 
225 inner plexiform layer (GCIPL) within a 10-mm diameter circular region of interest (ROI) centered

226 at the fovea (Figure 1A), a region which has been shown to be advantageous over the

227 commonly used 5-mm diameter ROI for mRNFL measurements. ${ }^{37}$ It also reports peripapillary

228 RNFL (pRNFL) thickness at a 3.45-mm diameter circle centered at the ONH (Figure 1B). We

229 further subdivided pRNFL into temporal, superior, nasal, and inferior. We additionally acquired

230 two $6 \times 6 \mathrm{~mm}$ OCT-A scans centered at the fovea (Figure 1C). We required an image quality of

$2317 / 10$ or better, as well as the absence of any artifacts or segmentation errors for all OCT and

232 OCT-A scans, resulting in the exclusion of 9 out of the 105 subjects.

After registering and binarizing the signal of the en face OCT-A images, we calculated

234 the fractal dimension (FD) of the superficial vascular plexus, inside a 3-mm diameter circle

235 centered at the fovea (Figure 1C). We have previously provided details on FD and its

236 calculation, as well as on the specifications and repeatability of the Canon OCT-A. ${ }^{28,38}$ In short,

237 FD represents the complexity of the branching pattern and is lower in conditions with sparser

238 vasculature, such as glaucoma. ${ }^{39}$

239 Lastly, we acquired two $45^{\circ}$ high-quality and artifact-free fundus images (TRC-NW400,

240 Topcon Corporation, Tokyo, Japan), centered at the ONH (Figure 1D). For each image, we

241 derived the central retinal artery and vein equivalents (CRAE, CRVE; i.e., diameters) using the

242 standardized Knudtson-Parr-Hubbard iteration, whose details and validation can be found

243 elsewhere. ${ }^{28,40}$ In short, we back-calculated vessel diameters using the 6 largest arteriolar and 6

244 largest venular branches, identified within a ring centered at the $\mathrm{ONH}$ (2 and 3 optic disc

245 diameters). We recorded the average CRAE and CRVE of two images.

247 Retinal blood flow and lower autoregulation limit

We calculated total retinal vascular resistance (RVR) using the measured FD, CRAE,

250 and CRVE of each participant, as well as population-based hematocrit values (Lifelines 
251 Biobank), adjusted for age, sex, and blood pressure status. We have previously documented

252 the mathematics behind this Poiseuille-based model and its validation in vivo. ${ }^{28,41}$ Subsequently,

253 we computed total RBF, using RVR and retinal perfusion pressure (RPP), a more precise

254 estimation of OPP for the retinal circulation ${ }^{28}$ :

$256 \quad \mathrm{RBF}=\frac{\mathrm{RPP}}{\mathrm{RVR}}$

257 where $\mathrm{RPP}=(0.39 \cdot \mathrm{MAP}+10.1)-\mathrm{IOP} \mathrm{mmHg}$ and $\mathrm{MAP}=\frac{1}{3} \mathrm{SBP}+\frac{2}{3} \mathrm{DBP}$ is the mean arterial 258 pressure.

We defined LARL as the lowest RPP value for which RBF can be maintained constant

261 (Figure 2). At this critical point, the vasculature has reached its maximal autoregulatory capacity

262 and any further pressure drop will not trigger compensatory vasodilation, resulting in flow

263 reduction. We have previously shown that $L A R L$ can be approximated as:

266 where $\mathrm{RVR}_{\max }$ is an upper bound observed in a population. ${ }^{28}$

268 In this study, we defined $R V R_{\max }$ as the 95th percentile of the RVR distribution. Due to the

269 possible occurrence of structural remodeling in retinal vessels belonging to subjects with $\mathrm{AHT}$,

270 we separated the RVR distributions of the non-hypertensives (groups 1 and 2) and

271 hypertensives (groups 3 and 4). ${ }^{12}$

272 Lastly, for each participant, we defined the autoregulatory reserve (AR) as the difference

273 between measured RPP and predicted LARL (Figure 2). 
For the first part of the analysis, to establish the existence of a U-shaped association (if 279 any), we univariably compared structural OCT metrics (mRNFL, GCIPL, and pRNFL) between

280 the four BP groups. For the second part of the analysis, to investigate whether any vascular 281 factors could possibly explain this association, we univariably compared RVR, RBF, and AR 282 between the groups. For the last part of the analysis, we performed mediation analysis to 283 examine whether the vascular metrics lie in the explanatory pathway of the relationship between 284 MAP and the structural OCT metrics that were significant in the first part of the analysis.

one-way ANOVA with post hoc tests for group mean comparisons, adjusting for potential correction, or quantile regression.

294 the following were true in linear regression analysis:

a) $\mathrm{X}$ was a significant predictor of $\mathrm{Y}(\mathrm{Y} \sim \mathrm{X})$.

b) $\mathrm{X}$ was a significant predictor of $\mathrm{M}(\mathrm{M} \sim \mathrm{X})$.

299 We verified these findings by using the Sobel test for indirect effects. Mediation analysis was 300 performed separately for the low BP group together with the controls, the treated AHT group 
301 together with the controls, and the untreated AHT group together with the controls. RBF, RVR,

302 and AR were examined as potential mediators.

$303 \quad$ All analyses were performed using R (version 3.3.3; R Foundation for Statistical

304 Computing, Vienna, Austria) and SPSS (version 26; IBM Corp., Armonk, NY). A P value of 0.05

305 or less was considered statistically significant.

306

307 Results

308

In total, 96 eyes of 96 subjects fulfilled all the criteria and were included in the analysis.

310 Table 1 displays the characteristics of the population, stratified by BP status. Sex and BMI were

311 significantly different between groups, aside from BP. As expected, the low BP group comprised

312 almost exclusively females, while higher BMI was present in the hypertensive groups. ${ }^{43,44}$ Other

313 factors that could affect the comparisons, such as age, IOP, spherical equivalent (SEQ), and

$314 \mathrm{ONH}$ area, were similar between groups.

316 Table 1. Characteristics of the study population.

\begin{tabular}{|c|c|c|c|c|c|}
\hline & $\begin{array}{l}\text { Group } 1 \\
\text { (Low BP) }\end{array}$ & $\begin{array}{c}\text { Group } 2 \\
\text { (Normal BP) }\end{array}$ & $\begin{array}{c}\text { Group } 3 \\
\text { (Treated AHT) }\end{array}$ & $\begin{array}{c}\text { Group } 4 \\
\text { (Untreated AHT) }\end{array}$ & \multirow{2}{*}{$P$ value } \\
\hline Group size $(N)$ & 31 & 21 & 26 & 18 & \\
\hline $\begin{array}{l}\text { Age; years } \\
\text { [mean (SD)] }\end{array}$ & $56.1(4.4)$ & $55.9(4.7)$ & $56.4(4.8)$ & $57.2(4.6)$ & 0.81 \\
\hline Sex; \% female & 93.5 & 47.6 & 42.3 & 44.4 & $<0.001$ \\
\hline $\begin{array}{l}\text { SBP; mmHg } \\
\text { [mean (SD)] }\end{array}$ & $106(9)$ & $126(6)$ & $142(18)$ & $159(22)$ & $<0.001$ \\
\hline $\begin{array}{l}\text { DBP; mmHg } \\
\text { [mean (SD)] }\end{array}$ & $66(6)$ & $79(6)$ & $86(11)$ & $99(8)$ & $<0.001$ \\
\hline
\end{tabular}




\begin{tabular}{|c|c|c|c|c|c|}
\hline $\begin{array}{l}\text { BMI; kg·m-2 } \\
\text { [median (IQR)] }\end{array}$ & $\begin{array}{c}22.1 \\
(21.2 \text { to } 24.3)\end{array}$ & $\begin{array}{c}23.3 \\
\text { (22.1 to } 26.5)\end{array}$ & $\begin{array}{c}26.9 \\
(24.7 \text { to } 29.8)\end{array}$ & $\begin{array}{c}27.3 \\
(24.3 \text { to } 28.4)\end{array}$ & $<0.001$ \\
\hline Smoking; \% ever & 22.6 & 38.1 & 30.8 & 38.9 & 0.57 \\
\hline $\begin{array}{l}\text { IOP; mmHg } \\
\text { [mean (SD)] }\end{array}$ & $13.9(3.0)$ & $13.2(3.1)$ & $14.3(3.0)$ & $14.6(3.7)$ & 0.56 \\
\hline $\begin{array}{l}\text { SEQ; D } \\
\text { [mean (SD)] }\end{array}$ & $-0.10(1.41)$ & $+0.27(1.67)$ & $-0.23(1.55)$ & $-0.68(1.69)$ & 0.31 \\
\hline $\begin{array}{l}\text { ONH area; } \mathrm{mm}^{2} \\
\text { [median (IQR)] }\end{array}$ & $\begin{array}{c}1.89 \\
(1.69 \text { to } 2.24)\end{array}$ & $\begin{array}{c}1.96 \\
(1.71 \text { to } 2.20)\end{array}$ & $\begin{array}{c}1.94 \\
(1.72 \text { to } 2.31)\end{array}$ & $\begin{array}{c}2.00 \\
(1.78 \text { to } 2.29)\end{array}$ & 0.75 \\
\hline \multicolumn{6}{|c|}{$\begin{array}{l}\text { BP, blood pressure; } \mathrm{AHT} \text {, arterial hypertension; SD, standard deviation; SBP, systolic blood pressure; } \\
\text { DBP, diastolic blood pressure; BMI, body mass index; IOP, intraocular pressure; SEQ, spherical } \\
\text { equivalent; ONH, optic nerve head. }\end{array}$} \\
\hline
\end{tabular}

\section{Structural metrics}

Table 2 and Figure 3 present the comparison of structural OCT metrics across the four

321 BP groups. Adjusted post hoc comparisons revealed that, compared to the controls, GCIPL was

322 significantly thinner in the low BP group $\left(P_{a d j}=0.013\right)$, the treated AHT group $\left(P_{a d j}=0.007\right)$, and

323 the untreated AHT group $\left(P_{a d j}=0.007\right)$. The mRNFL was also thinner, but this was only

324 significant for the treated AHT group $\left(P_{a d j}=0.001\right)$. Interestingly, mRNFL in treated

325 hypertensives was even significantly thinner than in untreated hypertensives $\left(P_{\text {adj }}=0.033\right)$.

326 Figure 3 shows the characteristic (inverse) U shape for the macular OCT metrics. There was no

327 clear effect of BP group on the mean pRNFL. However, treated hypertensives had a thinner

328 temporal pRNFL $\left(P_{a d j}=0.045\right)$ than normotensives. Also, inferior pRNFL was borderline thinner

329 in subjects with low BP $\left(P_{a d j}=0.083\right)$ and clearly thinner in both treated and untreated

330 hypertensives $\left(P_{a d j}=0.034\right.$ and 0.033 , respectively). Sex and BMI did not confound any of the 
332 the analysis.

334 Table 2. Structural OCT metrics as a function of BP status.

\begin{tabular}{|c|c|c|c|c|c|}
\hline & $\begin{array}{l}\text { Group } 1 \\
\text { (Low BP) }\end{array}$ & $\begin{array}{c}\text { Group } 2 \\
\text { (Normal BP) }\end{array}$ & $\begin{array}{c}\text { Group } 3 \\
\text { (Treated AHT) }\end{array}$ & $\begin{array}{c}\text { Group } 4 \\
\text { (Untreated AHT) }\end{array}$ & $P$ value \\
\hline $\begin{array}{l}\text { GCIPL; } \mu \mathrm{m} \\
\text { [mean (SD)] }\end{array}$ & $53.6(2.7)$ & $56.2(3.0)$ & $53.4(3.3)$ & $53.1(2.5)$ & 0.002 \\
\hline $\begin{array}{l}\text { mRNFL; } \mu \mathrm{m} \\
\text { [mean (SD)] }\end{array}$ & 37.7 (3.3) & $39.8(3.4)$ & $36.0(2.8)$ & $38.7(3.4)$ & 0.001 \\
\hline $\begin{array}{l}\text { Total pRNFL; } \mu \mathrm{m} \\
\text { [mean (SD)] }\end{array}$ & $99.4(8.2)$ & $103.1(10.1)$ & $96.9(9.2)$ & $100.3(8.8)$ & 0.14 \\
\hline $\begin{array}{l}\text { Temporal pRNFL; } \mu \mathrm{m} \\
\text { [mean (SD)] }\end{array}$ & $71.8(10.5)$ & $74.0(8.1)$ & 65.5 (9.5) & $72.6(15.3)$ & 0.038 \\
\hline $\begin{array}{l}\text { Superior pRNFL; } \mu \mathrm{m} \\
\text { [mean (SD)] }\end{array}$ & $119.6(14.7)$ & $124.1(16.7)$ & $119.5(13.5)$ & $126.1(16.6)$ & 0.36 \\
\hline $\begin{array}{l}\text { Nasal pRNFL; } \mu \mathrm{m} \\
\text { [mean (SD)] }\end{array}$ & $82.2(11.2)$ & $80.6(14.1)$ & $81.4(11.4)$ & $80.6(14.0)$ & 0.96 \\
\hline $\begin{array}{l}\text { Inferior pRNFL; } \mu \mathrm{m} \\
\text { [mean (SD)] }\end{array}$ & $124.5(12.9)$ & $134.0(13.3)$ & $122.7(14.3)$ & $121.5(15.7)$ & 0.019 \\
\hline \multicolumn{6}{|c|}{$\begin{array}{l}\text { OCT, optical coherence tomography; BP, blood pressure; } \mathrm{AHT} \text {, arterial hypertension; GCIPL, ganglion } \\
\text { cell-inner plexiform layer; SD, standard deviation; mRNFL, macular retinal nerve fiber layer; } \mathrm{PRNFL,} \\
\text { peripapillary retinal nerve fiber layer. }\end{array}$} \\
\hline
\end{tabular}

Vascular metrics

Figure 4 displays RBF, RVR, and AR as a function of BP status. There were differences

339 in RBF between groups $(P=0.034)$, but after adjusting for multiple comparisons RBF was only 
significantly lower in the low BP group when compared to the untreated AHT group $\left(P_{a d j}=\right.$ 0.043). RVR was also different between groups $\left(P=9.0 \cdot 10^{-9}\right)$, with the additional presence of a

342 larger variance in the treated AHT group (Levene's test: $P=0.002$ ). With regards to AR, the 343 unequal variances were also statistically significant (Levene's test: $P=0.0002$ ), showing that,

344 unlike any other group, treated hypertensives could have either a large or a small AR. As can be

345 better seen in Figure 5 and Supplementary Table S1, the low BP group had a significantly

346 smaller AR than the control group, regardless of AR quantile compared. Conversely, the

347 untreated hypertensives had a significantly larger AR than the control group, regardless of

348 quantile compared. However, there was a mixed response in the treated AHT group: the AR

349 was significantly smaller than that of the controls for small quantiles, while it was similar or

350 larger for larger quantiles. In addition, correlation analysis within the treated AHT group revealed

351 that the smaller AR quantiles corresponded to the lowest MAP values (Pearson's $r=0.45, P=$

352 0.020), i.e., to the most intensively controlled hypertensives. Again, sex and BMI did not

353 confound these associations.

Mediation analysis

Results from mediation analysis regarding the effect of BP status on GCIPL are

presented in Table 3. RBF was mediating the association of GCIPL with BP within the combined low BP group and controls, while RVR was mediating the same association within the combined untreated AHT group and controls. RVR and AR were both independently mediating the

361 association of GCIPL with BP within the combined treated AHT group and controls. In the

362 complete model (GCIPL MAP+RVR+AR), which accounts for the covariance between RVR and $363 \mathrm{AR}$, the opposite, real effect of AR became visible, that is, small AR was associated with thinner 364 GCIPL (see Discussion section). We did not observe any vascular mediation for the effect of BP 365 status on RNFL metrics. 
367 Table 3. Effect of BP status on GCIPL: mediation analysis.

\begin{tabular}{|c|c|c|c|c|c|c|}
\hline & \multicolumn{2}{|c|}{$\begin{array}{l}\text { Low BP + controls } \\
\text { (Group } 1 \text { + Group 2) }\end{array}$} & \multicolumn{2}{|c|}{$\begin{array}{c}\text { Controls + treated AHT } \\
\text { (Group } 2+\text { Group } 3 \text { ) }\end{array}$} & \multicolumn{2}{|c|}{$\begin{array}{c}\text { Controls + untreated AHT } \\
\text { (Group } 2+\text { Group 4) }\end{array}$} \\
\hline & Effect & $P$ value & Effect & $P$ value & Effect & $P$ value \\
\hline \multicolumn{7}{|l|}{ Mediation step 1: } \\
\hline GCIPL MAP & $b_{M A P}=0.09$ & 0.052 & $b_{\text {MAP }}=-0.10$ & 0.043 & $b_{M A P}=-0.07$ & 0.032 \\
\hline \multicolumn{7}{|l|}{ Mediation step 2: } \\
\hline RBF MAP & $b_{M A P}=0.27$ & 0.020 & $N / A$ & $N / A$ & $N / A$ & $N / A$ \\
\hline RVR MAP & $N / A$ & $N / A$ & $b_{M A P}=0.005$ & 0.008 & bMAP $=0.005$ & 0.0003 \\
\hline AR MAP & $N / A$ & $N / A$ & $\mathrm{~b}_{\mathrm{MAP}}=0.17$ & 0.015 & $N / A$ & $N / A$ \\
\hline \multicolumn{7}{|l|}{ Mediation step 3: } \\
\hline \multirow{2}{*}{ GCIPL MAP + RBF } & $\mathrm{b}_{\mathrm{MAP}}=0.05$ & 0.30 & $N / A$ & $N / A$ & $N / A$ & $N / A$ \\
\hline & $b_{\mathrm{RBF}}=0.16$ & 0.003 & $N / A$ & $N / A$ & $N / A$ & $N / A$ \\
\hline \multirow{2}{*}{ GCIPL MAP + RVR } & $N / A$ & $N / A$ & $b_{\text {MAP }}=-0.04$ & 0.42 & $b_{M A P}=-0.03$ & 0.51 \\
\hline & $N / A$ & $N / A$ & $b_{\text {RVR }}=-11.87$ & 0.001 & $b_{R V R}=-10.23$ & 0.022 \\
\hline \multirow{2}{*}{ GCIPL MAP + AR } & $N / A$ & $N / A$ & $b_{M A P}=-0.06$ & 0.21 & $N / A$ & $N / A$ \\
\hline & $N / A$ & $N / A$ & $b_{A R}=-0.21$ & 0.042 & $N / A$ & $N / A$ \\
\hline \multirow{3}{*}{ GCIPL $\sim \mathrm{MAP}+\mathrm{RVR}+\mathrm{AR}$} & $N / A$ & $N / A$ & $b_{M A P}=-0.04$ & 0.40 & $N / A$ & $N / A$ \\
\hline & $N / A$ & $N / A$ & $b_{\text {RVR }}=-27.37$ & 0.001 & $N / A$ & $N / A$ \\
\hline & $N / A$ & $N / A$ & $b_{A R}=0.47$ & 0.032 & $N / A$ & $N / A$ \\
\hline Sobel test & \multicolumn{2}{|c|}{0.059} & \multicolumn{2}{|c|}{ RVR: 0.041; AR: 0.095} & \multicolumn{2}{|c|}{0.031} \\
\hline
\end{tabular}


$\mathrm{BP}$, blood pressure; GCIPL, ganglion cell-inner plexiform layer; AHT, arterial hypertension; MAP, mean arterial pressure; RBF, retinal blood flow; RVR, retinal vascular resistance; $A R$, autoregulatory reserve; $N / A$, not applicable.

Discussion

In this study, we reported three main findings. Firstly, there exists an inverse U-shaped effect between blood pressure status and structural OCT metrics (GCIPL and RNFL), with both low and high blood pressure being associated with thinning of the inner retinal layers. Secondly,

374 despite the existence of retinal blood flow autoregulation, only a small autoregulatory reserve is present in individuals with low blood pressure, as well as in individuals with intensively treated arterial hypertension. Lastly, this compromised capacity for retinal blood flow regulation explains

377 (mediates) the effect of blood pressure status on the GCIPL.

Low blood pressure

This is, to our knowledge, the first study to uncover an association between low BP and

382 thinning of the inner retina in ophthalmologically healthy subjects. This relationship and its 383 vascular mediation were more pronounced for the GCIPL, which has been shown to be the 384 main layer of early NTG manifestation. ${ }^{45}$ In addition, the association was entirely mediated by 385 RBF (no effect of sex, BMI, or other confounders in our population).

Indeed, we have previously shown that LARL for subjects without AHT corresponds to a 387 realistic SBP/DBP of $\sim 105 / 65 \mathrm{mmHg}$ (or even higher if IOP is above average)..$^{28}$ Since, in the 388 present study, the average BP reading for the low BP group was at $106 / 66 \mathrm{mmHg}$, our finding 389 that this group had a borderline lower RBF (Figure 4, Table 3) and a considerably smaller AR 
390 (Figure 5, Table S1) than controls is in line with our estimations and the general concept of 391 autoregulation.

Population studies have failed to report this association between low BP and GCIPL thickness, possibly due to the implementation of linear models, but an explanation due to

394 differences in genetic background cannot be excluded. ${ }^{33,34}$ However, nonlinear models were 395 used is studies with glaucoma as the outcome measure and, in line with our findings, there is 396 evidence for the existence of increased glaucoma risk with low, usually diastolic or nocturnal, 397 BP. ${ }^{13-16,18,46}$

\section{Hypertension} hypertensives had thinner GCIPL, which has also been shown to be the location of first progression in glaucoma patients with AHT. ${ }^{45}$ This was mediated by RVR (Table 3), i.e., the negative effect of increased BP to the GCIPL is explained by increased vascular resistance (but not by reduced blood flow - see below). Nevertheless, it was the treated AHT group that exhibited the most pronounced thinning and this was present in the majority of structural OCT metrics (GCIPL, mRNFL, temporal pRNFL, inferior pRNFL). For this group, GCIPL thinning was

408 independently mediated by RVR and AR (Figure 5, Table 3). In the univariable models, large

409 RVR and large AR were both associated with thinner GCIPL, due to substantial covariance.

410 After controlling for the confounding effect of RVR, small AR was associated with thinner

411 GCIPL. This suggests that, in treated AHT, a combination of increased resistance and being

412 close to the autoregulatory tipping point explain the negative effect to the GCIPL.

413 GCIPL thinning without a decrease in RBF seems counterintuitive. However, even when

414 total RBF is largely unaffected, increased RVR results in increased blood velocity (i.e., reduced

415 transit time), shunting of flow, and reduced capillary density. ${ }^{47,48}$ This could affect red blood cell 
416 distribution and retinal oxygen extraction. Smaller AR was additionally present in intensively

417 treated subjects (Figure 4C, Figure 5, Table S1), which could mimic low BP and lead to

418 hypoperfusion of the RGCs. In this regard, our results reflect a possible effect of the combined

419 rightward shift of the autoregulation curve (due to atherosclerosis and arteriosclerosis) and the

420 variations of the measured RPP (Figure 2), due to BP fluctuations throughout the day. Lastly,

421 chronic AHT also results in endothelial dysfunction and, therefore, impaired autoregulation. ${ }^{49}$

422 A number of previous studies have also shown an effect of AHT on GCIPL and RNFL

423 thickness. ${ }^{21,29-32}$ However, some population-based studies did not detect this relationship. ${ }^{33,34}$

424 Again, study design and analysis methods seem to be the most likely explanations for this

425 discrepancy. In all, the existence of thinner GCIPL in both low BP and AHT creates the

426 characteristic inverse U-shaped association. Regarding glaucoma risk, the role of AHT is

427 controversial. Most evidence points towards at least some benefit of timely AHT treatment,

428 possibly due to the prevention of microvascular damage, in combination with a slight IOP

429 lowering. ${ }^{24,50}$ However, it has been suggested that aggressive treatment of AHT could

430 negatively affect glaucoma and our results also point towards this direction. ${ }^{20}$ The confounding

431 contribution of individual antihypertensive medications, whether neuroprotective or detrimental,

432 remains inconclusive. ${ }^{21-25}$

433 We would like to stress here that, since there are benefits to intensive BP control with

434 regards to cardiovascular disease, our results should not be considered as a case for milder

435 treatment of AHT in general. ${ }^{51,52}$ However, since a $\mathrm{J}$ or U-shaped effect is reported in both fields

436 when intensive treatment becomes too intensive, our results could be a starting point for

437 discussion with cardiologists in individual cases where, for example, glaucoma continues to

438 deteriorate despite adequate IOP control. ${ }^{53}$

440 ‘Chicken-egg’ dilemma 
medRxiv preprint doi: https://doi.org/10.1101/2021.01.14.21249808; this version posted January 15, 2021. The copyright holder for this preprint (which was not certified by peer review) is the author/funder, who has granted medRxiv a license to display the preprint in perpetuity.

It is made available under a CC-BY-NC-ND 4.0 International license .

From a theoretical standpoint, there exists a point in the pre-disease time course when

443 the very first vascular deficits or the very first structural deficits manifest. A subsequent causal

444 cascade of events would then result in further mutually mediated vascular and structural

445 deterioration, sometimes leading to a glaucoma diagnosis. By definition, cross-sectional studies

446 regarding perfusion in glaucoma cases are well past this critical point and cannot enhance our

447 understanding of the process.

$448 \quad$ In this regard, our study's novelty lies in demonstrating that interdependent structural

449 and vascular deficits related to a long-debated cardiovascular risk factor (especially low BP) can

450 even be traced back to whom we perceive as ophthalmologically healthy subjects. These results

451 suggest that a subtle GON precursor remains rather elusive, albeit present early on. While this

452 observation adequately explains the claim that perfusion deficits are already present prior to

453 glaucoma, it does not fully resolve the 'chicken-egg' dilemma (we showed that vascular deficits

454 are present without glaucoma, but not necessarily without smaller structural deficits). However,

455 the existence of different mediators between MAP and GCIPL thickness with different BP status,

456 hints towards a preceding vascular component. For example, there is no parsimonious

457 explanation for an intensive antihypertensive treatment strategy following GCIPL thinning.

Study strengths and limitations

The main strength of this study was the strict selection process which allowed us to look

462 at the true extremes of BP. This reduces the noise that usually characterizes larger population

463 studies and results in indirect loss of power. In addition, our linearity-free assumptions and the

464 categorizing of BP (rather than considering it as a continuous variable) allowed us to

465 differentiate between BP status and uncover a U-shaped association that was previously

466 elusive. Lastly, to our knowledge, this study is the first to provide a rigorous explanation of the 
medRxiv preprint doi: https://doi.org/10.1101/2021.01.14.21249808; this version posted January 15, 2021. The copyright holder for this preprint (which was not certified by peer review) is the author/funder, who has granted medRxiv a license to display the preprint in perpetuity.

It is made available under a CC-BY-NC-ND 4.0 International license .

467 differential effect of BP status on retinal structure, by directly linking it to total RBF and its 468 autoregulation.

469 Due to the cross-sectional nature of our study, absence of data on the first occurrence of

470 AHT is a limitation. However, our threshold of an at least 1-year old diagnosis, together with the

471 selection procedure using multiple previous visits from another database, ensured no newly-

472 diagnosed cases (almost all cases had been diagnosed before at least 3 years). Secondly,

473 RBF, RVR, and AR were not directly measured, but indirectly calculated by means of their

474 measured components and a previously validated model. Indeed, we have previously shown in

475 an independent validation dataset that these outcomes strongly correlate with in vivo blood flow

476 metrics, as well as with structural metrics. ${ }^{28}$ Unfortunately, there is currently no gold standard

477 way to quantify these parameters, but Doppler OCT is a frequently-used, promising tool. Our

478 predictive approach has the advantage of using more reproducible imaging techniques and,

479 most importantly, taking into account autoregulation limits. Combining these two methods could,

480 therefore, further finetune estimations. It should be also noted that our approach provides

481 information on the effect of static RBF autoregulation, but it is possible that BP status also

482 results in impairment of the autoregulatory latency, i.e., dynamic autoregulation. As such, our

483 results might only be part of a bigger underlying effect. Lastly, our population was predominantly

484 Caucasian; it is to be determined if the results can be generalized to other ethnicities.

In conclusion, we uncovered thinning of the GCIPL and RNFL related to both tails of the

487 blood pressure distribution (inverse U-shaped effect) and to intensive treatment of $\mathrm{AHT}$, in

488 ophthalmically healthy individuals. We found that GCIPL thinning was associated with reduced

489 autoregulatory capacity of retinal blood flow. This predisposition to glaucomatous damage could

490 explain the frequent epidemiological finding of increased glaucoma risk in certain population

491 subgroups, such as subjects with nocturnal blood pressure dipping or aggressively treated

492 hypertension. Longitudinal studies are needed to confirm this postulation. 

and glaucomatous eyes using optical coherence tomography. Arch Ophthalmol. 1995;113(5):586596. randomised, multicentre, placebo-controlled trial. Lancet. 2015;385(9975):1295-1304. progression: results from the Early Manifest Glaucoma Trial. Arch Ophthalmol. 2002;120(10):12681279. 1972;56(3):229-242. Res. 2002;21(4):359-393. $1991 ; 254(5035): 1178-1181$. coherence tomography. Opt Express. 2012;20(4):4710. imaging by Fourier domain optical coherence tomography. J Biomed Opt. 2002;7(3):457. Ophthalmology. 2016;123(12):2498-2508. 2021;105(1):124-130. structure measurements in glaucoma. Invest Ophthalmol Vis Sci. 2012;53(6):3020-3026. 
Res. 2001;20(5):595-624.

13. Leske MC, Heijl A, Hyman L, et al. Predictors of long-term progression in the early manifest glaucoma trial. Ophthalmology. 2007;114(11):1965-1972. incident open-angle glaucoma: the Barbados Eye Studies. Ophthalmology. 2008;115(1):85-93. Blood pressure, perfusion pressure, and open-angle glaucoma: the Los Angeles Latino Eye Study. Invest Ophthalmol Vis Sci. 2010;51(6):2872-2877. perfusion pressure and the incidence of glaucoma: real effect or artifact? The Rotterdam Study. Invest Ophthalmol Vis Sci. 2011;52(9):6875-6881. Glaucomatous Optic Neuropathy-A Systematic Review and Meta-Analysis. American Journal of Hypertension. 2015;28(9):1077-1082. glaucoma: a meta-analysis. Am J Ophthalmol. 2014;158(3):615-627.e9. pressure status in the Thessaloniki Eye Study. Am J Ophthalmol. 2013;155(5):843-851. Nerve Fiber Layer and Ganglion Cell-Inner Plexiform Layer Thickness. Ophthalmology. 2020;31:S0161-6420(20)30739-9. doi:10.1016/j.ophtha.2020.07.051 incident open-angle glaucoma. Ophthalmology. 2007;114(12):2221-2226. Uncontrolled Primary Open-Angle Glaucoma. Ophthalmology. 2018;125(7):984-993.

24. Horwitz A, Klemp M, Jeppesen J, Tsai JC, Torp-Pedersen C, Kolko M. Antihypertensive 
Medication Postpones the Onset of Glaucoma: Evidence from a Nationwide Study. Hypertension. 2017;69(2):202-210. with Glaucoma Progression and Glaucoma Suspect Conversion in the Groningen Longitudinal Glaucoma Study. Invest Ophthalmol Vis Sci. 2019;60(14):4548-4555. in Glaucoma Patients: The Lifelines Cohort Study. Invest Ophthalmol Vis Sci. 2020;61(11):25. with laser Doppler velocimetry. Invest Ophthalmol Vis Sci. 1986;27(12):1706-1712. autoregulation range in the human retina: in vivo investigation with Laser Speckle Flowgraphy. Am J Physiol Heart Circ Physiol. 2020;319(6):H1253-H1273. doi:10.1152/ajpheart.00404.2020 NERVE FIBER, AND GANGLION CELL INNER PLEXIFORM LAYERS IN PATIENTS WITH HYPERTENSION. Retina. 2019;39(9):1810-1818. Layer Thickness and Retinal Microvasculature in Hypertension: An Optical Coherence Tomography Angiography Study. Am J Ophthalmol. 2019;199:167-176. systemic arterial hypertension: an OCT study. Eur J Ophthalmol. 2016;26(5):436-441. thickness and its association with carotid intima media thickness. Blood Press. 2015;24(3):178-184. Multi-Ethnic Asian Population. Ophthalmology. 2020;127(8):1064-1076. 2012;53(9):5853. 500. 

Speckle Flowgraphy and Doppler Optical Coherence Tomography. Invest Ophthalmol Vis Sci. 2016;57(13):5417-5425.

37. Heikka T, Cense B, Jansonius NM. Retinal layer thicknesses retrieved with different segmentation algorithms from optical coherence tomography scans acquired under different signalto-noise ratio conditions. Biomed Opt Express. 2020;11(12):7079. Assessed by Optical Coherence Tomography Angiography. Trans/ Vis Sci Technol. 2019;8(3):3. summarizing retinal vessel diameters. Curr Eye Res. 2003;27(3):143-149. hemodynamic parameters in the human retinal microvascular network. J Biorheol. 2009;23(2):77-86. research: conceptual, strategic, and statistical considerations. J Pers Soc Psychol. 1986;51(6):11731182.

43. Tigchelaar EF, Zhernakova A, Dekens JAM, et al. Cohort profile: LifeLines DEEP, a prospective, general population cohort study in the northern Netherlands: study design and baseline characteristics. BMJ Open. 2015;5(8):e006772. Vliet-Ostaptchouk JV. Sex, BMI and age differences in metabolic syndrome: the Dutch Lifelines Cohort Study. Endocr Connect. 2017;6(4):278. 

Spontaneously Hypertensive Rats. Invest Ophthalmol Vis Sci. 2020;61(10):12. using optical coherence tomographic angiography. J Hypertens. 2019;37(3):572-580. function of the retinal vasculature in hypertensive patients. Stroke. 2004;35(6):1289-1293. disease and death: a systematic review and meta-analysis. Lancet. 2016;387(10022):957-967. target systolic blood pressure (120-140 $\mathrm{mmHg}$ ) and cardiovascular outcomes in high-risk patients: 


\section{Figure 1.}

630 Structural (A-B) and vascular (C-D) regions of interest. A) Macular retinal nerve fiber layer

631 (mRNFL) and ganglion cell-inner plexiform layer (GCIPL) thicknesses measured within the 632 larger (10-mm diameter) circle centered at the fovea, excluding the innermost 1-mm diameter 633 circle and the nasal sector of the outer ring. B) Peripapillary RNFL (pRNFL) measured at a circle 634 of 3.45-mm diameter centered at the optic nerve head (ONH). C) Optical Coherence

635 Tomography-Angiography (OCT-A) scan of $6 \times 6 \mathrm{~mm}$ centered at the fovea. Signal intensity 636 inside the innermost 3-mm diameter circle is binarized in flow (black) and non-flow (white). D)

$63745^{\circ}$ fundus image centered at the $\mathrm{ONH}$. The 6 largest arterioles and 6 largest venules between 638 rings of 2 and 3 optic disc diameters are marked in blue.

Figure 2.

642 Theoretical autoregulation curve (axes in arbitrary units). Retinal blood flow (RBF) is displayed 643 as a function of retinal perfusion pressure (RPP). Within the autoregulation range, blood flow is 644 maintained constant. The distance of the actual (measured) RPP from the lower autoregulation 645 limit $(L A R L)$ is the autoregulatory reserve (AR).

\section{$647 \quad$ Figure 3.}

649 Ganglion cell-inner plexiform layer (GCIPL), macular retinal nerve fiber layer (mRNFL), and

650 peripapillary retinal nerve fiber layer (pRNFL) as a function of blood pressure (BP) status.

651 Significant differences after post hoc comparisons (adjusted for multiple testing) are marked.

652 The thicker layers observed in the control group (normal BP) than in the low BP or arterial 653 hypertension (AHT) groups create a characteristic (inverse) $U$ shape. 


\section{Figure 4.}

657 Absolute total retinal blood flow (RBF), retinal vascular resistance (RVR), and autoregulatory

658 reserve $(A R)$ as a function of blood pressure (BP) status. Significant differences after post hoc

659 comparisons (adjusted for multiple testing) are marked. With increasing BP, RBF increases less

660 than RVR, resulting in an autoregulation effect visible in the first panel. A statistically significant

661 larger variability is observed for the AR of treated hypertensives (third panel), suggesting that

662 subjects in this group can be very close or very far from the lower autoregulation limit.

\section{Figure 5.}

667 Parameter estimates (y-axis) represent the relative AR (compared to controls) for every AR

668 decile (x-axis). Low BP individuals (left panel) have a smaller AR than controls $(\mathrm{y}<0)$, while

669 individuals with untreated arterial hypertension (AHT; right panel) have a larger AR than controls

$670(y>0)$. Individuals with treated AHT (middle panel) have a smaller AR only when intensively

671 treated (leftmost deciles). 
medRxiv preprint doi: https://doi.org/10.1101/2021.01.14.21249808; this version posted January 15, 2021. The copyright holder for this preprint (which was not certified by peer review) is the author/funder, who has granted medRxiv a license to display the preprint in perpetuity.

It is made available under a CC-BY-NC-ND 4.0 International license .

Figure 1

681

682

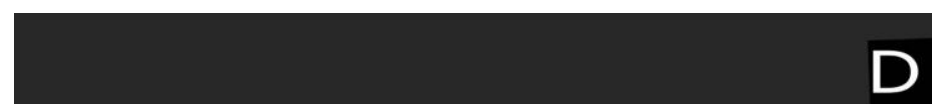

683

684

A

Figure 2

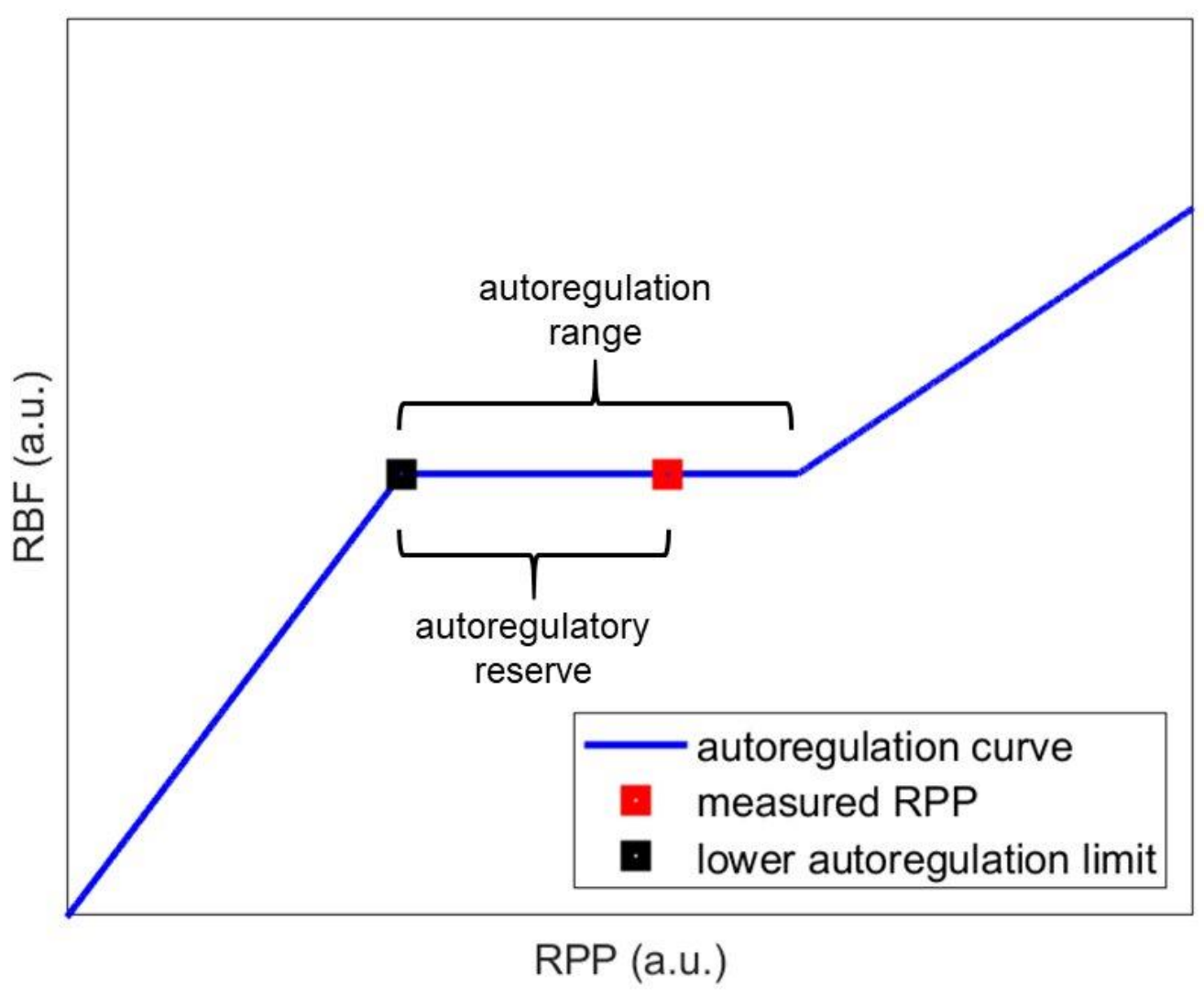


medRxiv preprint doi: https://doi.org/10.1101/2021.01.14.21249808; this version posted January 15, 2021. The copyright holder for this preprint (which was not certified by peer review) is the author/funder, who has granted medRxiv a license to display the preprint in perpetuity.

\section{It is made available under a CC-BY-NC-ND 4.0 International license .}

\section{Figure 3}

687
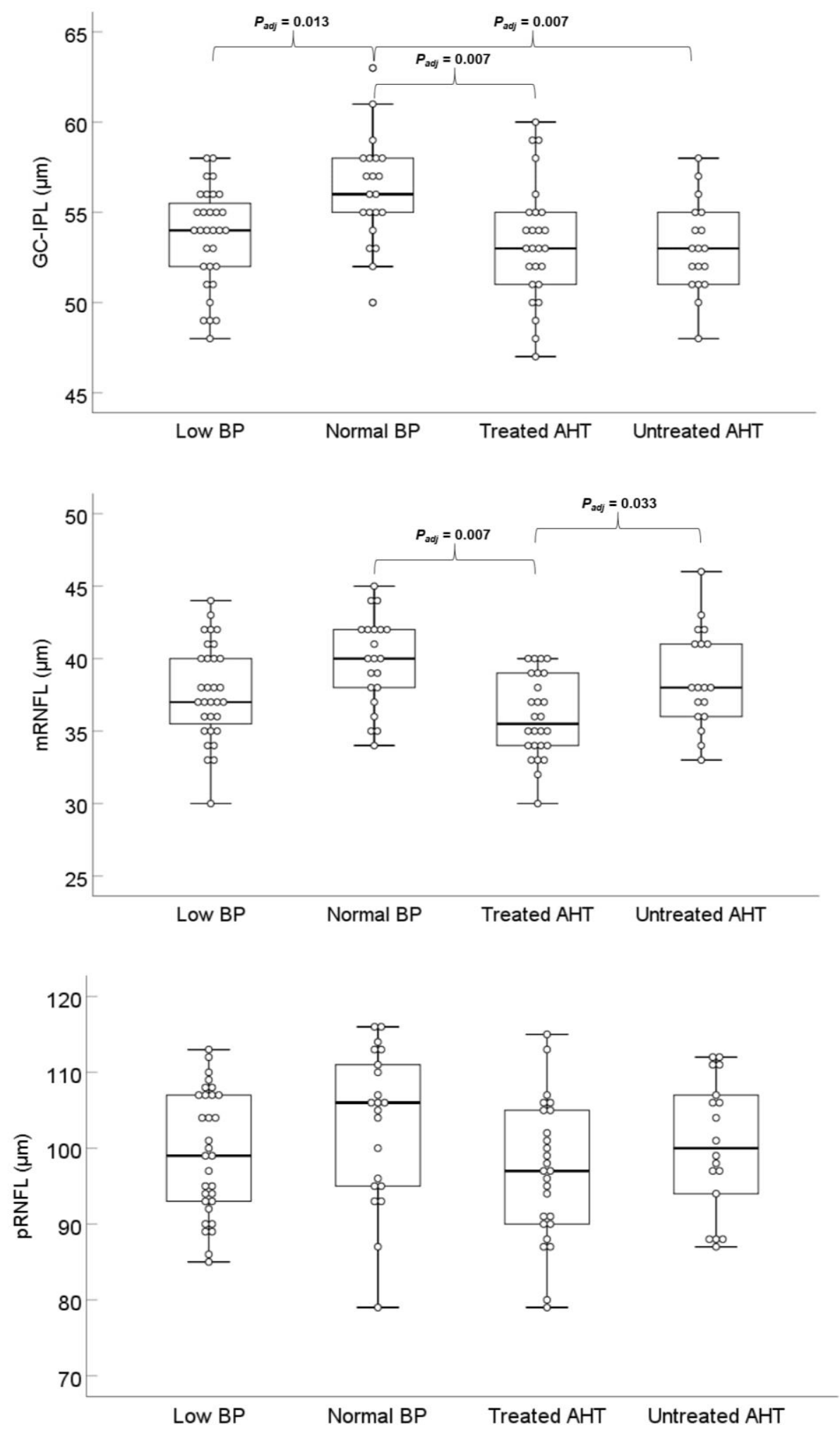
medRxiv preprint doi: https://doi.org/10.1101/2021.01.14.21249808; this version posted January 15, 2021. The copyright holder for this preprint (which was not certified by peer review) is the author/funder, who has granted medRxiv a license to display the preprint in perpetuity.

\section{It is made available under a CC-BY-NC-ND 4.0 International license .}

\section{Figure 4}

690
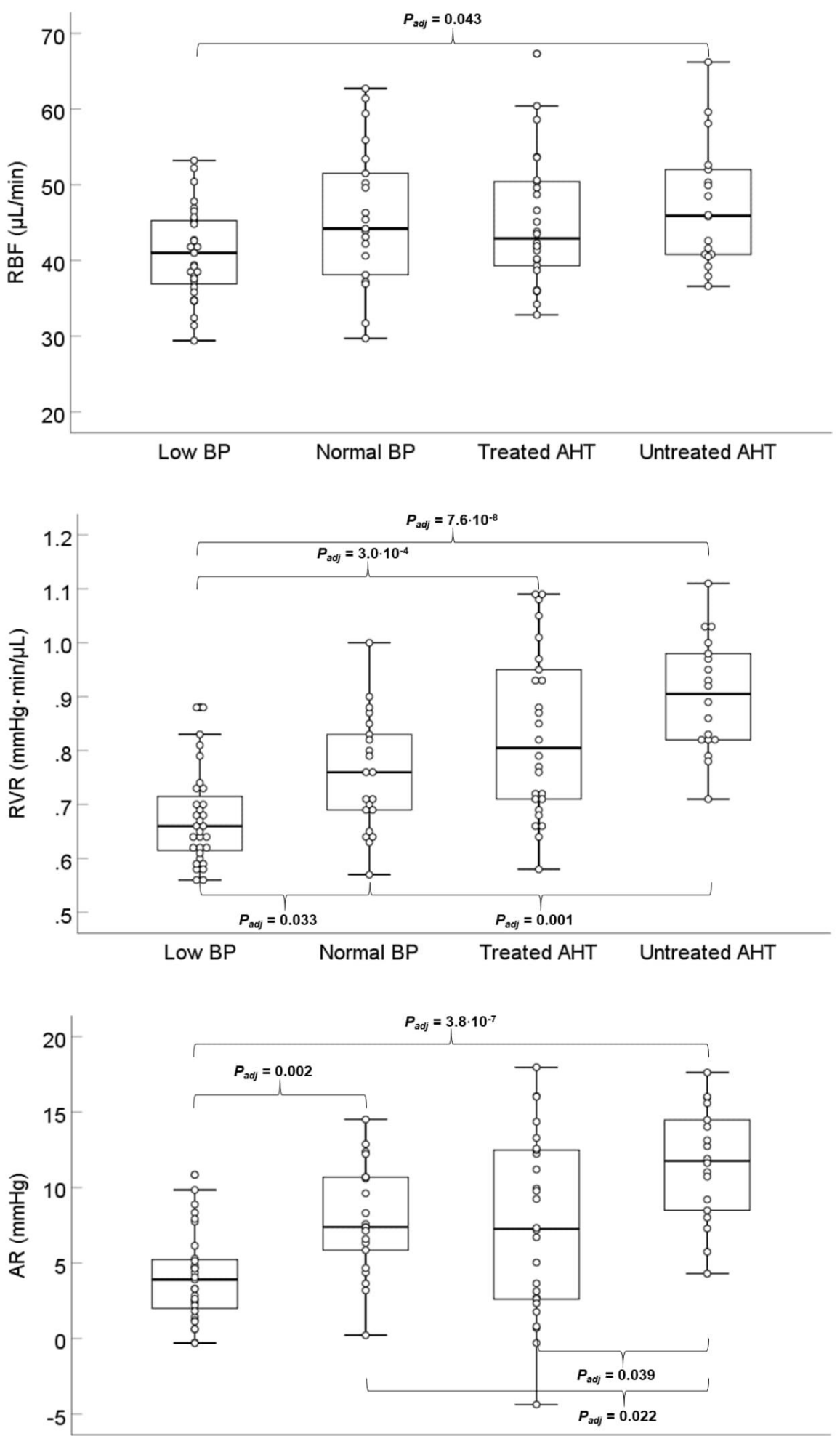

Low BP

Normal BP

Treated AHT Untreated AHT

691 
medRxiv preprint doi: https://doi.org/10.1101/2021.01.14.21249808; this version posted January 15, 2021. The copyright holder for this preprint (which was not certified by peer review) is the author/funder, who has granted medRxiv a license to display the preprint in perpetuity.

\section{It is made available under a CC-BY-NC-ND 4.0 International license .}

693

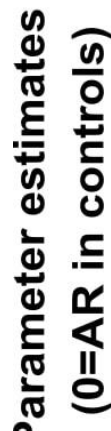

Q
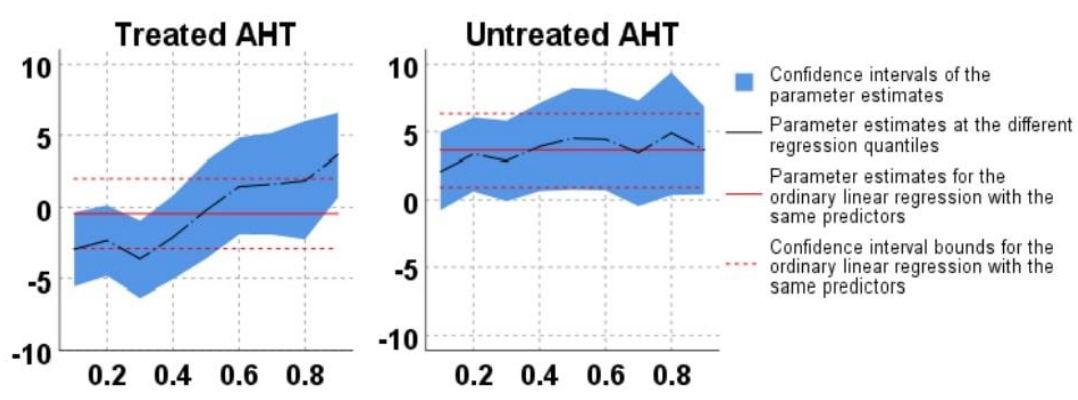